\title{
Pre-operative Assessment of Ovarian Tumor in Patients Presenting with Adnexal Mass on the Basis of Risk of Malignancy Index (RMI)
}

\author{
Chowdhury $\mathrm{S}^{1}$, Jahan $\mathrm{R}^{2}$, Hossain $\mathrm{DL}^{3}$, Sharmin $\mathrm{F}^{4}$, Rahman $\mathrm{S}^{5}$, Dewan $\mathrm{F}^{6}$,
}

Conflict of Interest: None
Financial Support: None
Received: $27-09-2017$
Accepted: $16-11-2017$
www.banglajol.info/index.php/JSSMC
Key Words:

Adnexal mass, Menopausal

status, RMI.

\begin{abstract}
Background:Ovarian cancer is the second most common gynaecologic cancer in developing countries. Five-year survival of ovarian cancer varies according to stages, hence, screening, detection and treatment in earlier stages are of great importance. The Scottish Intercollegiate Guidelines Network (SIGN) recommends use of Risk of Malignancy Index (RMI) for preoperative assessment of ovarian tumours. It is a scoring system based on menopausal status, ultrasound findings, and serum CA 125 level in adnexal mass.
\end{abstract}

Objective:To investigate the accuracy of RMI as a predictive method of discriminating benign from malignant ovarian tumour.

Methodology: Fifty patients, 30 years or older, with adnexal mass, admitted for laparotomy in Obstetrics and Gynaecology Dept. from April'13 to September'13were randomly assigned in this cross-sectional descriptive study. RMI was calculated for each patient based on menopausal status, CA125 level and ultrasound findings of bilateral lesion, multilocular cyst, solid areas, ascites and metastases.

Results:Among 50 women, 18 cases (36\%) were postmenopausal showing more malignant tumors (77.78\%) in this group. Depending on histopathological reports, $82 \%$ benign and $18 \%$ malignant diseases. The best performance of RMI was obtained at cut-off value of 230 with sensitivity of $100 \%$, specificity of $95.2 \%$, positive predictive value (PPV) of $80 \%$ and negative predictive value (NPV) $100 \%$.

Conclusion:Compared to previous studies, RMI was highly sensitive in detecting malignant disease, though not as specific in excluding benign lesions, particularly endometriosis.

[J Shaheed Suhrawardy Med Coll 2017; 9(2): 69-73] DOI: http://dx.doi.org/10.3329/jssmc.v9i2.37266

\section{Introduction}

Adnexal mass refers to any mass occupying the region of uterine appendages including ovarian neoplasm,

1. Dr. Sanjukta Chowdhury, Registrar, Department of Obstetrics \& Gynaecology, Shaheed Suhrawardy Medical College Hospital, Dhaka, Bangladesh.

2. Dr. Raunak Jahan, Consultant, Department of Obstetrics \& Gynaecology, Shaheed Suhrawardy Medical College Hospital, Dhaka, Bangladesh.

3. Dr. Dina Layla Hossain, Registrar, Department of Obstetrics \& Gynaecology, Dhaka Medical College Hospital, Dhaka, Bangladesh.

4. Dr. Fahmida Sharmin Joty, Assistant Professor, Department of Obstetrics \& Gynaecology, Care Medical College Hospital, Dhaka, Bangladesh.

5. Dr. Sumana Rahman, Junior Consultant, Department of Obstetrics and Gynaecology, Government Employees' Hospital, Fulbaria, Dhaka

6. Prof. Dr. Farhana Dewan, Professor \& Head of the Department of Obstetrics \& Gynaecology, Shaheed Suhrawardy Medical College Hospital, Dhaka, Bangladesh.

Correspondence to: Dr. Sanjukta Chowdhury,Registrar, Department of Obstetrics \&Gynaecology, Shaheed Suhrawardy Medical College Hospital, Sher-E-Bangla Nagar, Dhaka, Bangladesh; Email: sanjuktak58dmc@gmail.com; Cell No.: +8801816269329 endometrioma, tubo-ovarian mass, myoma, ectopic pregnancy ${ }^{1}$. Ovarian tumor is the second most common gynaecologic cancer variant in developing countries causing huge mortality among women ${ }^{2}$. Peak incidence of epithelial ovarian cancer (EOC) is at 50-70 years subsequently declining to less than $2 \%$ after 80 years. About $30 \%$ of ovarian tumors in post menopausaland $7 \%$ in pre-menopausal are malignant ${ }^{3.95 \%}$ of women with ovarian cancer have non-gynaecological symptoms like abdominal distension, bloating, constipation, nausea, anorexia, or early satiety ${ }^{4-7}$. Standard management of EOC consists of aggressive surgical cytoreduction followed by chemotherapy. Extensive retrospective experience showed that optimal surgical debulking with no or less than $1 \mathrm{~cm}$ residual tumor is associated with improved patient outcome ${ }^{8}$. Considering diagnostic difficulties, RMI, an acronym for risk of malignancy index, was first described by Jacobs et al in 1990, to identify high risk patients in order to triage them accurately to specialist care. It is the product of menopausal status, trans abdominal ultrasound 
(TAS) score and serum CA125 (U/mL). During TAS, appearance and distribution of tumor, ascites, solidity is noted $^{9}$. CA-125, a high molecular weight glycoprotein, is expressed by a large proportion of EOC. It is a wellestablished tumor marker for EOC and have an important role in diagnosis ${ }^{10}$. However, sensitivity and specificity of this marker is poor. It is raised in approximately $50 \%$ of stage I EOC and in $75-90 \%$ of advanced diseases ${ }^{11-16}$. In menstruating women CA-125 may be elevated in many benign entities like fibroid, adenomyosis, endometriosis. Butin post-menopausal women, CA-125 is a good diagnostic tool, as their quiescent ovaries and endometrium do not secret such proteins ${ }^{17}$.Jacobs I et al (1990) first applied RMI index among 143 patients with pelvic masses in London Hospital. In his study using RMI cut-off level of 200, sensitivity and specificity were $85 \%$ and $97 \%$ respectively. Patients with RMI score $>200$ had, on average, 42 times more risk of cancer and those with a lower value 0.15 times risk ${ }^{9}$. Hakanson et al have shown in their study that out of 1159 patients, there were 778 women had benign pelvic mass, 251 had malignant ovarian tumour and 74 had borderline variety. 56 patients were diagnosed with other forms of cancer. Sensitivity and specificity for ovarian cancer vs. benign pelvic mass for RMI > 200 were 92 and $82 \%$, respectively. Corresponding positive and negative predictive values were 62 and $97 \%{ }^{8}$.

\section{Methodology}

This cross-sectional descriptive study was conducted at the Department of Obstetrics and Gynaecology of ShSMCH from April 01, 2013 to September 30, 2013.Fifty women, 30-75 years, admitted with adnexal mass (diagnosed either per abdominally or sonographically) for laparotomy, were randomly selected. Those who have suspected uterine malignancy or deny surgery are excluded. After taking informed consent, menopausal status, serum CA125 level and ultrasound findings of participants were registered. If there were no bilateral lesions, multilocular cyst, solid areas, ascites and abdominal metastases on TAS, it was termed as no abnormality $(\mathrm{U}=1)$, if any one abnormality was found it was termed as one abnormality $(\mathrm{U}=1)$ and $>$ one abnormality was named as two $(\mathrm{U}=4)$. Laparotomy followed by histopathological analysis of specimens were done. RMI calculation was based on a simple regression equation where premenopausal status gave $\mathrm{M}=1$ and postmenopausal M=4. CA125 level was applied directly into calculation. All statistical analyses were done in SPPS inc.version 11.5. Chi-square tests were used to test difference in age distribution, menopausal status and ultrasound score. Sensitivity, Specificity, Positive
Predictive value (PPV) and Negative predictive value (NPV) were calculated for 3 cut-off values of RMI. Scoring should be carried out before laparotomy in assessing nature of mass. The best cut-off value was chosen according to the highest sensitivity with lowest false-positive rate.

\section{Results:}

Majority of the patients in this study were between 30-75 years with mean age being 40.7 years (SD 11.16 yrs) consisting of $32(64 \%)$ pre-menopausal and 18 (36\%) postmenopausal.

Table-I

Age distribution of patients $(n=50)$

\begin{tabular}{lcc} 
Age distribution & Frequency & Percentage $(\%)$ \\
\hline $30-39$ years & 28 & 56 \\
$40-49$ years & 10 & 20 \\
$50-59$ years & 10 & 20 \\
$60-75$ years & 02 & 04 \\
\hline Total & 50 & 100 \\
\hline
\end{tabular}

Abdominal pain was complained by all patients followed by lump in $32(64 \%)$, dyspepsia in 7 (14\%) and constipation in $5(10 \%)$ of patients. No abnormality on TAS was detected in $60 \%$, one abnormality in $20 \%$ and two or more abnormalities in another $20 \% .20$ patients (40\%) serum CA 125 fell within normal range ( $<35 \mathrm{U} / \mathrm{ml})$.Pre-operative RMI value for assessment was taken as 200 and found $76 \%$ patients' RMI $<200$ and $24 \%$ had $>200$. Among 10 categories of histopathologically diagnosed masses, majority occupied two domains ( $28 \%$ serous cyst adenoma, $24 \%$ endometriosis). Benign conditions included: functional cysts $(n=02)$, simple serous cyst $(n=14)$, Dermoid cyst $(\mathrm{n}=04)$, Endometriosis $(\mathrm{n}=12)$, Mucinous cystadenoma $(n=04)$, ectopic pregnancy $(n=02)$ and parovarian cyst $(\mathrm{n}=04)$ whereas malignant tumors: Serous cystadenocarcinoma $(\mathrm{n}=2)$, Mucinous cystadenocarcinoma $(n=2)$, and secondary adenocarcinoma $(n=4)$.
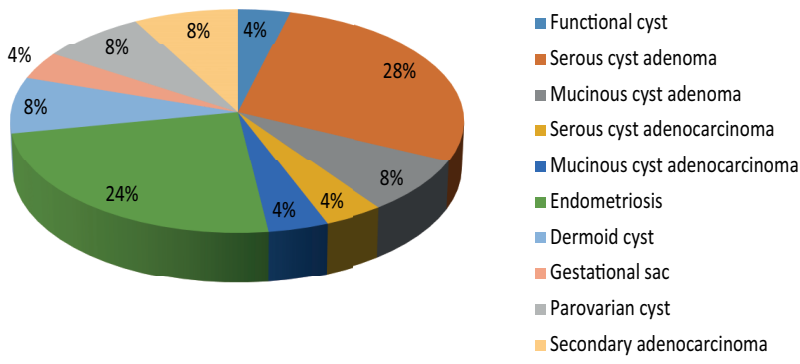

Fig.-1: Pie chart showing histopathological variants of adnexal masses 
Though statistically insignificant differences were found between groups with benign and malignant pathology considering age ( $\mathrm{P}$ value 0.074$)$, menopausal status ( $\mathrm{P}$ value 0.004), TAS score (P value .000) and CA 125 (Pvalue .000), it was shown that higher proportions of malignant cases were associated with higher age, US score and serum CA 125. The best performance was obtained for RMI cut-off value of 230 with sensitivity $100 \%$, specificity $95 \%$, PPV $80 \%$ and NPV $100 \%$.

\section{Table-II}

Sensitivity, specificity, positive predictive value (PPV) and negative predictive value (NPV) for RMI

RMI cut-off value Sensitivity Specificity PPV NPV

\begin{tabular}{lcccc}
\hline 150 & $100 \%$ & $90 \%$ & $71.4 \%$ & $100 \%$ \\
200 & $100 \%$ & $90.5 \%$ & $66.7 \%$ & $100 \%$ \\
230 & $100 \%$ & $95.2 \%$ & $80 \%$ & $100 \%$ \\
\hline
\end{tabular}

2 serous cyst adenomas along with 2 endometriomas at RMI 200 and 2 serous cystadenomas at RMI cut off value 230 were recorded as false positive with no false negative cases in both RMI.

\section{Discussion:}

Among 50 women of this study, 64\% were premenopausal. There were more malignant tumors (77.78\%) in postmenopausal group $(\mathrm{P}=0.004)$ suggestive of cancer incidence rises with increasing age and in postmenopausal group. Result was similar with studies of Tahereh et al 63.2\%, WatcharadaMoolthiya et al $60.8 \%$ and Samir et al 70\% $17,18,19$.

In the study $100 \%$ patients presented with abdominal pain and $64 \%$ with abdominal lump showing similar result by TayyibaWasim et al where abdominal pain was seen in $66 \%$ patients $^{20}$. In a retrospective cohort study by Lataifeh et al with, abdominal pain and swelling were reported by $51 \%$ and $32 \%$ respectively in early stage and lump by $62 \%$ and pain by $44 \%$ in advanced stage 7 . This study do not differentiate symptoms among early and advanced cases.

In $80 \%$ cases USG revealed only unilateral adnexal mass scoring 1 . All patients with $\mathrm{U}=1$ had benign tumors; on the other hand, $90 \%$ of them who obtained $U=4$ were malignant in histopathology. Though there was a difference between these two groups ( $p$ value 0.000 ), it was not statistically significant. Tahereh et al ${ }^{17}$ also showed, $98 \%$ patients with $U$ score $=1$ had benign tumors with $65.4 \%$ patients with high score had malignancy and it

Table-III

Distribution of age, menopausal status, ultrasound score and S. CA125 in 50 women with benign (n=41) and malignant $(n=09)$ adnexal mass.

\begin{tabular}{|c|c|c|c|}
\hline Age (yrs) & Benign & Malignant & $P$ value \\
\hline $30-39$ & $26(63.41 \%)$ & $02(22.22 \%)$ & \\
\hline $40-49$ & $07(17.07 \%)$ & $03(33.33 \%)$ & $0.074^{\mathrm{a}}$ \\
\hline $50-59$ & $06(14.63 \%)$ & $04(44.44 \%)$ & \\
\hline $60-75$ & $02(4.88 \%)$ & $0(0 \%)$ & \\
\hline \multicolumn{4}{|l|}{ Menopausal status } \\
\hline Pre menopausal & $30(73.17 \%)$ & $02(22.22 \%)$ & $0.004^{b}$ \\
\hline Post menopausal & $11(26.83 \%)$ & $07(77.78 \%)$ & \\
\hline \multicolumn{4}{|l|}{ Ultrasound score } \\
\hline 1 & $40(97.56 \%)$ & $0(0 \%)$ & $.000^{\mathrm{c}}$ \\
\hline 4 & $01(2.44 \%)$ & $09(100 \%)$ & \\
\hline S. CA125 value $(\mathrm{u} / \mathrm{ml})$ & Benign & Malignant & $P$ value \\
\hline$>35$ & $20(48.78 \%)$ & $0(0 \%)$ & \\
\hline $35-100$ & $15(36.58 \%)$ & $01(11.11 \%)$ & $.000^{\mathrm{d}}$ \\
\hline $101-200$ & $04(9.75 \%)$ & $02(22.22 \%)$ & \\
\hline $201-400$ & $02(4.89 \%)$ & $0(0 \%)$ & \\
\hline$>400$ & $0(0 \%)$ & $06(66.67 \%)$ & \\
\hline
\end{tabular}

$\mathrm{a}=4$ cells $(50 \%)$ have expected count less than 5 . The minimum expected count is 36 .

$\mathrm{b}=1$ cell $(25 \%)$ have expected count less than 5 . The minimum expected count is 3.24 .

$\mathrm{c}=2$ cells $(33.3 \%)$ have expected count less than 5 . The minimum expected count is 1.80 .

$\mathrm{d}=8$ cells $(80 \%)$ have expected count less than 5 . The minimum expected count is .36 . 
Table-IV

\begin{tabular}{lccccc}
\multicolumn{7}{c}{ Comparison of our Results with Previous Studies* } \\
Study & No. of cases & Sensitivity & Specificity & PPV & NPV \\
\hline Jacobs et al.1990 & 143 & 85.4 & 96.9 & & \\
Davies et al.1993 & 124 & 87.0 & 89.0 & & \\
Tingulstad et al. 1996 & 173 & 71.0 & 96.0 & 89 & 88 \\
Manjunath et al. 2001 & 152 & 73.0 & 91.0 & 93.0 & 67.0 \\
Andersen et al. 2003 & 180 & 70.6 & 87.7 & 66 & 90 \\
Obeidat et al. 2004 & 100 & 90.0 & 89.0 & 96 & 78 \\
Ulusoy et al. 2006 & 296 & 71.7 & 80.5 & 67 & 84 \\
Tehereh et al. 2009 & 1159 & 89.5 & 94.7 & 71 & 98 \\
Hakanson et al. 2012 & 159 & 92 & 82 & 62 & 97 \\
Samir et al. 2011 & 140 & 86.67 & 92.5 & 89.66 & 90.24 \\
Present study (RMI 230) & 50 & 100 & 95.2 & 80 & 100 \\
\hline
\end{tabular}

*Values were $\%$ given for RMI=200; PPV, positive predictive value; NPV, negative predictive value

was also statistically insignificant. Association of higher sonographic score with malignant cases was also supported by WatcharadaMoolthiya et al and Samir et al. Serum CA1 25 value $>200 \mathrm{u} / \mathrm{ml}$ associated with malignant conditions was also supported by previous studies ${ }^{17,18}$. Main limitation of serum CA125 is its high value in benign diseases such as ovarian cysts, endometriosis and pelvic infection ${ }^{21}$, reflected in present study. In this study out of 50 patients with adnexal mass, based on histopathological reports, less malignant cases $(18 \%)$ were diagnosed than benign which is in concordance with previous studies 34,36 . WatcharadaMoolthiya et al, stated in his study majority false positive cases were due to dermoid cysts and mucinous cyst adenomas while false negative cases were for borderline tumors, result not supported by this study ${ }^{18}$.

At lower cut off values sensitivity increases at the expense of specificity, while at a higher cut off values specificity increases at the expense of sensitivity and more benign cases will be referred as malignant. These findings are important for clinical applicability of RMI. In the present study sensitivity of RMI (100\%) to predict malignancy was higher than those reported by the previous studies $^{10,9,17,18,22,23}$. One possible explanation is that we cannot differentiate early stage and borderline ovarian tumor.

\section{Conclusion}

This study demonstrated that RMI scoring is a simple and effective one in assessing nature of adnexal masses preoperatively, despite some limitations. It guides the clinicians to determine which tumors will be operated by
Gynaecologists/Gynae-oncologist and thereby improves patient's outcome.

\section{Acknowledgements}

I would like to express my heartfelt gratitude to my respected guide Professor Dr. FarhanaDewan, former Head, Dept. of Obstetrics \& Gynaecology, ShSMCH and all my co-authors for their immense help and valuable suggestions.

\section{References}

1. Dutta D.C. Special topics - Adnexal Mass in Textbook of Gynaecology. $5^{\text {th }}$ d, Kolkata New Central Book Agency Ltd.2008:543.

2. Greenlee et al., 2001. CA Cancer J Clin. 2001 Jan-Feb; 51(1):15-36. Cancer statistics, 2001.

3. Sully RE, Young RH, Clement PB, Atlas of Tumor pathology 1998.

4. Friedman GD, Skilling JS, Udaltsova NV, Smith LH. Early symptoms of ovarian cancer: a case control study without recall bias. Fam Pract 2005; 22; 548-53.

5. Smith LH, Morris CR, Yasmeen S, Parikh-Patel A, Cress RD, Romano PS. Ovarian cancer: can we make the clinical diagnosis earlier? Cancer 2005; 104:1398 - 407.

6. Koldjeski D, Kirkpatrick MK, Swanson M, Everett I, Brown S. Ovarian cancer: early symptom patterns. OncolNurs Forum 2003; 30: 927 - 33.

7. Lataifeh I, Marsden DE, Robertson G, Gebski V, Hacker NF . Presenting symptoms of epithelial ovarian cancer. Aust N Z J ObstetGynaecol 2005; 45: 2114.

8. Hankinson SE, Hunter DJ, Colditz GA, et al. Tubal ligation, hysterectomy, and risk of ovarian cancer. A prospective study. JAMA. 1993;270:2813-2818.

9. Jacobs I;OramD;FairbankJ;TurnerJ;FrostC;Grudzinskas JG. A risk of malignancy index incorporating CA125, Ultrasound 
and menopausal status for accurate preoperative diagnosis of ovarian cancer. Br.J.OB.Gyn.1990; 97:922-929.

10. Tingulstad S; Hagen B; Skjeldestad FE; OnscudM;Kiserud T Halvorsen T; Nustad K. Evaluation of a risk of malignancy index based on serum CA124,ultrasound findings and menopausal status in the pre-operative diagnosis of pelvic mass. Br.J.Ob.Gyn.1996; 103:826-831.

11. Ozguroglu M,Turna H, Demir G, et al. Usefulness of the epithelial tumour marker CA125 in non-Hodgkin's lymphoma. Am J ClinOncol1999;22:615-18.

12. Levine CD, Patel UJ, Ghanekar D, et al. Benign extraovarian mimics of ovarian cancer. Distinction with imaging studies. Clin Imaging 1997;21:350-8

13. Meden H, Fattahi-Meibodi A. CA125 in benign gynaecological conditions. Int J Biol Markers 1998;13: 231-7.

14. Gaspar MJ, Diez H, Rodriguez A, et al. Clinical value of CEA and $C A 125$ regarding relapse and metastasis in resectable non-small cell lung cancer. Anticancer Res2003;23:342732.

15. Krishnan STM, Philipose Z, Rayman G. Hypothyroidism mimicking intra-abdominal malignancy. BMJ 2002; 325:946-7.

16. Buamah P. Benign conditions associated with raised serum CA125 concentration. J SurgOncol2000;75:264-5.
17. TaherehAshrafgangooei*, MahdiehRezaeezadeh ,Risk of Malignancy Index in Preoperative Evaluation of Pelvic Masses, Asian Pacific J Cancer Prev, 12, 1727-1730.

18. WatcharadaMoolthiya, PissamaiYuenyao, The Risk of Malignancy Index (RMI) in Diagnosis of OvarianMalignancy, 2007, Asian Pacific J Cancer Prev, 10, 865-868

19. Samir Mohamed Fouad Abdel Aziz, MD, OBGYN.net Editorial Advisor, Ali Saleh, MD, Mohamed Said El-Shorbagy, MD,Preop Diagnosis of Ovarian Cancer in Patients Presented with Adnexal Mass, Obstetrics \& Gynecology Department, Oncology Department and clinical pathology Department Al-Azhar University, Cairo-Egypt September 23, 2011

20. TayyibaWasim,SaqibSiddiq ( Department of OBG, Services Institute of Medical Sciences) )Ashraf Majrroh ( Department of Community Medicine, Allama Iqbal Medical College, Lahore ),2009, Comparison of clinical presentation of Benign and Malignant Ovarian Tumours.

21. Einhorn N; Bast RC; Knapp RC; Tjernberg N; Zurawski VR. Preoperative evaluation of serum CA125 levels in patients with primary epithelial ovarian cancer. Obstet Gynecol 1986;67:414-416

22. Davis AP;Jacobs I; Wools R; Fish A; Oram D. The adenxalmass:benign or malignant? Evaluation of a risk of malignancy index. Br.J.Ob.Gyn.1993; 100:927-931.

23. Manjunath AP, Pratapkumar, Sujatha K, Vani R. Comparison of three Risk-of Malignancy Indices in evaluation of pelvic masses. GynecolOncol 2001. 